\title{
A News Analysis and Tracking System
}

\author{
Sk. Mirajul Haque, Lipika Dey, and Anuj Mahajan \\ TCS Innovation Labs, Delhi \\ \{skm.haque, lipika.dey, anuj.mahajan\}@tcs.com
}

\begin{abstract}
Continuous monitoring of web-based news sources has emerged as a key intelligence task particularly for Homeland Security. We propose a system for web-based news tracking and alerting. Unlike subscription-based alerts, alerting is implemented as a personalized service where the system is trained to recognize potentially important news based on user preferences. Preferences are expressed as combinations of topics and can change dynamically. The system employs Latent Dirichlet Allocation (LDA) for topic discovery and Latent Semantic Indexing (LSI) for alerting.
\end{abstract}

\section{Introduction}

The amount of news content available online is increasing at a steady rate. News belongs to a very specific genre of textual data. News contents from multiple sources are usually similar in that they largely look at the same set of events, yet they are at the same time highly unstructured and open ended. Timely assimilation and interpretation of information available from these sources is extremely important for political and economic analysts. It is imperative to analyze the whole collection to eliminate the possibility of missing anything.

Automated acquisition, aggregation and analysis of news content is a challenging and exciting line of research, lying at the crossroads of information extraction, machine learning, machine translation, pattern discovery, etc. The complexity of the problem increases due to the unpredictability of incoming content that needs to be analyzed. User preferences cannot be expressed as pre-defined categories. A news item of any category - be it political, sports, or entertainment can become interesting to an analyst, depending on its content. An analyst may wish to track it for some time. Further the interest changes dynamically. The key challenge in recognizing relevant news lies in recognizing the concepts of interest to a user.

In this paper, we have presented a system that is distinct in functionality from other news analysis systems. It is designed as a watch-guard which continuously scans online sources for new stories and alerts a registered user whenever there is any new development that could be of potential interest to the user. The news repository is organized topically. Users can register their interests with the system in terms of topics. The system generates an alert if a new story is judged to be conceptually relevant to a user. Alerts are also sent to the user's mobile. The novelty of the proposed system lies in its capability to track conceptually relevant news items as opposed to news in pre-specified categories. 


\section{News Analysis Systems - A Review}

News categorization and topic tracking through news collections has been an active area of research for a few years now. In [1] five categorization techniques were compared for news categorization. It was found that Support Vector Machines (SVM), kNearest Neighbor $(\mathrm{kNN})$ and neural networks significantly outperform Linear Least squares Fit (LLSF) and Naive Bayes (NB) when the number of training instances per category is small. [4] presents a system for automatic categorization of news stories written in Croatian language into a pre-defined set of categories using $\mathrm{kNN}$ based classifier. [10] described a news analysis system to classify and index news stories in real time. The system extracted stories from a newswire, parsed the sentences of the story, and then mapped the syntactic structures into a concept base.

Since news contents change in unpredictable ways supervised techniques that require large training sets are not ideally suited for news repository organization. Topic tracking and detection [2] is another area of research where the emphasis is to monitor online feed of news articles, and determine the underlying topics and their distribution in the news items in an unsupervised way. In [7] a topic tracking system based on unigram models was presented. Given a sparse unigram model built from topic training data and a large number of background models built from background material, the system finds the best mixture of background models that matches the unigram model. [8] proposed a clustering based approach to summarize and track events from a collection of news Web Pages. In [9] the problem of skewed data in topic tracking was tackled using semi-supervised techniques over a bi-lingual corpus. The Lydia project [3] describes a method for constructing a relational model of people, places and other entities through a combination of natural language processing and statistical analysis techniques.

\section{Overview of the Proposed News Analysis System}

Fig.1 presents the software architecture of the proposed news analysis system. The key components of the system are:-

- News acquisition module - The News acquisition module collects news from a host of pre-defined websites from their $\operatorname{RSS}^{1}$ feeds. The RSS feeds contain news headlines, date and time of publishing, a brief description, and the URL of the full news story. These are periodically updates. The URLs extracted from the RSS feeds are fed to customized web crawlers, to extract the news stories.

- Topic Discovery - This module builds a topic map for the entire news collection using Latent Dirichlet Allocation (LDA). It also indexes the collection topically. Each time a news repository is updated, the existing topic maps are backed up and a new topic map is built.

- Topic Tracker and Trend Analyzer - Topic maps are time-stamped. These can be compared to analyze how the strength of a topic has changed over time. Correlation analysis of topic maps identifies entry and exit of new topics into the system. Topic tracking and trend analysis can provide early warnings and actionable intelligence to avoid disasters.

\footnotetext{
${ }^{1}$ http://en.wikipedia.org/wiki/RSS_(file_format).
} 


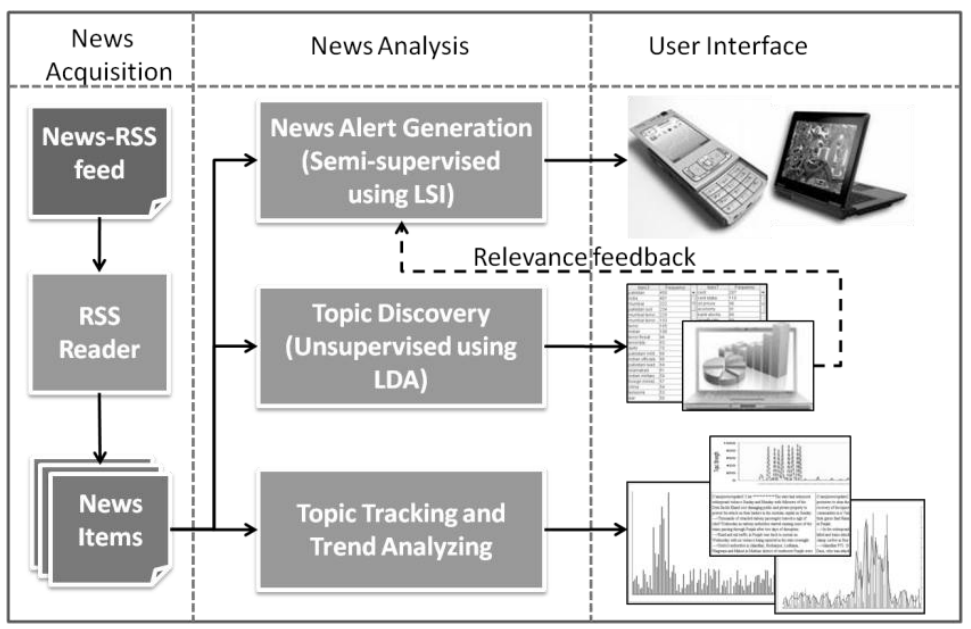

Fig. 1. Architecture of proposed News Analysis System

- News Alert Generator - This is a trainable module that implements Latent Semantic Indexing (LSI) to categorize an incoming news story as relevant or irrelevant to any of the multiple interest areas expressed by the user. The alerting mechanism is designed as a trainable multi-classifier system. User interests can be further classified as long-term and short-term. Concepts in long term interest category change at a slower rate than those in the short-term interest category. While long-term interests encode generic needs of a news monitoring agency, short-term interests help in tracking evolving stories in any category.

- Topic Explorer - This is the interface through which the user interacts with the system. A topic snap-shot shows all topics and concepts present in a collection. Users can drill-down to detailed reports on any event along any topic. This allows a single story to be read from multiple perspectives based on its contents. Topicbased aggregation allows the user to get a bird's eye view of the key events. Users can also view topic strengths and localizations.

\subsection{Topic Extraction Using LDA}

An important step to comprehend the content of any text repository is to identify the key topics that exist in the collection, around which the text can be organized. Latent Dirichlet Allocation (LDA) [5] is a statistical model, specifically a topic model, in which a document is considered as a mixture of a limited number of topics and each meaningful word in the document can be associated with one or more of these topics. A single word may be associated to multiple topics with differing weights depending on the context in which it occurs. Given a collection of documents containing a set of words, a good estimation of unique topics present in the collection is obtained with the assumption that each document $d$ can be viewed as a multinomial distribution over $k$ topics. Each topic $\mathrm{z}_{\mathrm{j}}, \mathrm{j}=1 \cdots k$, in turn, is assumed to be a multinomial distribution $\Phi(\mathrm{j})$ over the set of words $\mathrm{W}$. The problem of estimating the various distributions is in 
general intractable. Hence a wide variety of approximate algorithms, that attempt to maximize likelihood of a corpus given the model have been proposed for LDA. We have used the Gibb's sampling based technique proposed in [5] which is computationally efficient. The topic extractor creates a topic dictionary where each topic is represented as a collection of words along with their probabilities of belonging to the topic. Each document $d$ is represented as a topic distribution vector < $\left(\mathrm{p}\left(\mathrm{t}_{1}, d\right), \ldots, \mathrm{p}\left(\mathrm{t}_{\mathrm{k}}, d\right)>\right.$, where $\mathrm{p}\left(\mathrm{t}_{\mathrm{j}}, d\right)$ denotes the strength of topic $\mathrm{t}_{\mathrm{j}}$ in document $d$ is computed as follows:

$$
\mathrm{p}\left(\mathrm{t}_{\mathrm{j}}, \mathrm{d}\right)=\sum_{w=1}^{w=n} p\left(w, t_{j}\right) .
$$

where $n$ is the total number of unique words in the collection.

\subsection{News Alert Generation Using Latent Semantic Indexing}

All incoming news stories are scored for relevance based on its topic distribution. The system employs Latent Semantic Indexing (LSI) to assign relevance scores. LSI uses Singular Value Decomposition (SVD) to identify semantic patterns between words and concepts present in a text repository. It can correlate semantically related terms within a collection by establishing associations between terms that occur in similar context.

User interests are conveyed in terms of topics. Given that the underlying repository has stories containing different topics, of which some are relevant and some not, the aim is to find new stories which are conceptually relevant to the user, even though they may not be content-wise similar to any old story as such. The training documents for the system are created as follows. For each relevant topic, all stories containing the topic with strength greater than a threshold are merged to create a single training document $d_{R}$. All remaining stories are merged to create another training document $d_{I R}$. Let $T_{m 2}$ denote the term-document matrix for this collection, where $m$ represents the number of unique words. The weight of a word in $\mathrm{T}$ is a function of its probability of belonging to the topics in each category. Applying SVD to T yields,

$$
T=U W V^{T} \text {. }
$$

where $\mathrm{U}$ and $\mathrm{V}$ are orthonormal matrices and $W$ is a diagonal matrix. A modified term-document matrix $\widehat{T}$ is now constructed using $\widehat{U}$ which consists of the first two columns of $U$ such that $\widehat{T}$ represents a transformed 2 dimensional concept-space, where each dimension is expressed as a linear combination of the original terms and the relevant and irrelevant documents are well-separated. For each new news story, its cosine similarity with the relevant and irrelevant documents is computed in the new concept space. Let $Q$ represent the term vector for the new story. $Q$ is then projected into the transformed concept space to get a new vector $\hat{Q}$. Let $S_{R}$ and $S_{I R}$ denote the cosine similarity of $\hat{Q}$ with the relevant and irrelevant documents respectively. The new story is judged as relevant if

$$
S_{R}>\alpha * S_{I R}
$$

where $\alpha$ is a scaling parameter in the range $(0,1)$. Since number of news stories in irrelevant category is usually much higher than number of relevant news, there is an 
automatic bias towards the irrelevant category. The scaling parameter $\alpha$ adjusts this bias by reducing the similarity to irrelevant category.

\section{Experimental Results}

Fig. 2 presents some snapshots. On the left is a portion of the topic dictionary created for a 2 week time-period ending on $9^{\text {th }}$ June, 2009. Users select topics to indicate preferences for receiving alert. On the right an alert generated for the user is shown.

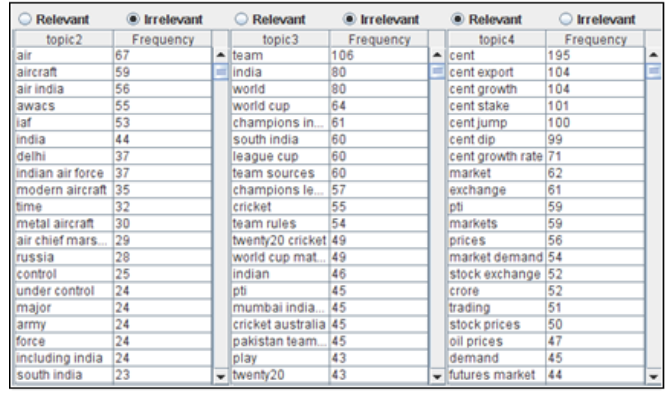

Fig. 2. Snapshots from the system

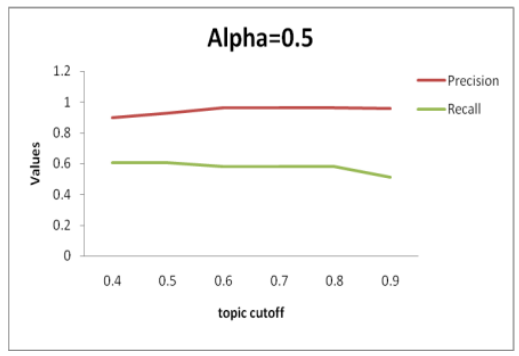

(a)

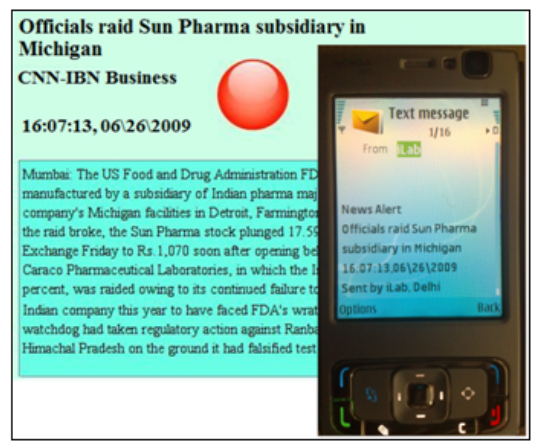

c.

Fig. 3. (a) Precision and Recall with varying topic cutoff and fixed alpha=0.5 (b) Precision and Recall with varying alpha and topic cutoff $=0.6$

Since user preferences are given as topics, it is observed that the accuracy of recognizing new stories as relevant depends on (a) the size of the training set (b) the scaling factor $\alpha$. Size of the training set is controlled using the topic cut off threshold. Fig. 3 shows system performance varies with these two parameters. With a fixed alpha, it is observed that recall falls slightly with higher values of topic cut-off, though precision does not suffer. Higher topic cut-off reduces the training set size causing the recall to fall. Sharper trends are observed with a fixed topic cut-off, and changing values of alpha. Recall falls drastically with higher values of alpha, since very few stories are now picked as relevant, though the precision is very high. Best results are observed for alpha and topic cut-off set to 0.5 and 0.6 respectively. 


\section{Conclusion}

In this work, we have presented a news analysis system which generates userpreference based alerts. Preferences are for topics and concepts rather than predefined categories. The news repository is analyzed and indexed topically. We are currently working on integrating a mining and analytical platform to this system to enhance its prediction and tracking capabilities.

\section{References}

1. Yang, Y., Liu, X.: A re-examination of text categorization methods. In: Proceedings of 22nd ACM SIGIR, California (1999)

2. Allan, J., Papka, R., Lavrenko, V.: On-line New Event Detection and Tracking. In: Proceedings of 21st ACM SIGIR, Melbourne (1998)

3. Lloyd, L., Kechagias, D., Skiena, S.: Lydia: A System for Large-Scale News Analysis. In: Consens, M.P., Navarro, G. (eds.) SPIRE 2005. LNCS, vol. 3772, pp. 161-166. Springer, Heidelberg (2005)

4. Bacan, H., Pandzic, I.S., Gulija, D.: Automated News Item Categorization. In: JSAI (2005)

5. Blei, D.M., Ng, A.Y., Jordan, M.I.: Latent Dirichlet Allocation. Journal of Machine Learning Research 3, 993-1022 (2003)

6. Landauer, T.K., Foltz, P.W., Laham, D.: An Introduction to Latent Semantic Analysis. Discourse Processes 25, 259-284 (1998)

7. Yamron, J.P., Carp, I., Gillick, L., Lowe, S., Van Mulbregt, P.: Topic Tracking in a News Stream. In: Proceedings of DARPA Broadcast News Workshop (1999)

8. Mori, M., Miura, T., Shioya, I.: Topic Detection and Tracking for News Web Pages. In: IEEE/WIC/ACM International Conference on Web Intelligence, pp. 338-342 (2006)

9. Fukumoto, F., Suzuki, Y.: Topic tracking based on bilingual comparable corpora and semisupervised clustering. ACM Transactions on Asian Language Information Processing 6(3) (2007)

10. Kuhns, R.J.: A News Analysis System. In: Proc. of 12th International Conference on Computational Linguistics, COLING 1988, vol. 1, pp. 351-355 (1988) 